\title{
Are stock returns an inflation hedge for the UK? Evidence from a wavelet analysis using over three centuries of data
}

\author{
${ }^{1}$ Center for Energy and Sustainable Development (CESD), Montpellier Business School, Montpellier, France \\ 2 University of Navarra, School of Economics, Edificio Amigos, E-31080 Pamplona, Spain \\ ${ }^{3}$ Department of Economics, University of Pretoria, Pretoria 0002, South Africa \\ ${ }^{4}$ College of Business Administration, University of Nebraska at Omaha, 6708 Pine Street, Omaha, NE 68182, USA, E-mail: \\ mwohar@unomaha.edu \\ ${ }^{5}$ School of Business and Economics, Loughborough University, Leicestershire, LE11 3TU, UK, E-mail: mwohar@unomaha.edu
}

\begin{abstract}
:
This paper analyzes the relationship between stock returns and the inflation rates for the UK over a long time period (February 1790-February 2017) and at different frequencies, by employing a wavelet analysis. We also compare the results for the UK economy with those for the US and two developing countries (India and South Africa). Overall, our results tend to suggest that, while the relationship between stock returns and inflation rates varies across frequencies and time periods, there is no evidence of stock returns acting as an inflation hedge, irrespective of whether we look at the two developed or the two developing markets in our sample.
\end{abstract}

Keywords: frequency-domain, inflation, nominal and real stock returns, wavelet analysis

JEL classification: C49, E31, G12

DOI: $10.1515 /$ snde-2017-0049

\section{Introduction}

While the 19th century was characterized by periods of moderate inflation and deflation in the UK (between 1790 and 1914, a period of over a century, prices were at about the same level), the evolution of inflation in the 20th and the beginning of the 21st centuries has been very different. First, as far as the inflation trend is concerned, since the First World War, prices have increased more than 40-fold (O'Donoghue, Goulding and Allen, 2004). Second, the period can be divided into a number of episodes of deflation (such as the interwar instability period from 1924 to 1939; the Bretton Woods and the Dollar Standard period from 1949 to 1970; or, the relatively more stable decades from 1980 to 2000) and inflation (the period after World War I, from 1914 to 1924; the World War II and postwar period, from 1939 to 1949; or, the oil price crisis period from 1973 to 1981, when prices more than tripled, with inflation reaching $24 \%$ in 1975 , and exceeding $10 \%$ in each year except 1978$),{ }^{1}$ showing the great heterogeneity in the inflation rate behavior over these years. Inflation rates have always been a key variable of interest, and its stabilization constitutes one of the objectives of the UK monetary policy. Although inflation decreases the value of money, according to the generalized Fisher hypothesis (Fisher 1930), in an efficient market, investors should be fully compensated for the increased price levels with an increase in nominal stock returns, so that real stock returns should only reflect expectations about real factors. This implies that real stock returns and inflation should vary independently, that is, stock returns should serve as a hedge against inflation, and, if this theory holds, we should observe a positive and one-to-one relationship between nominal stock returns and inflation rates. On the other hand, a positive relationship between nominal stock returns and inflation rates could also be explained by the Wealth Effect Hypothesis (Ando and Modigliani, 1963), since real stock returns can impact inflation rates through their effect on consumption and hence aggregate demand. According to this theory, there are different channels through which stock prices can affect consumption (see, for example, Ludwig and Sløk, 2004; Simo-Kengne et al., 2015), such as the realized gain, the expectation that raising the current stock price will result in higher future income and wealth, the liquidity constraint effect and the stock option value effect. Based on these two theories (Fisher Effect and Wealth Effect), a positive relationship between nominal stock returns and inflation rates should be observed in the data. 
The empirical relationship between (nominal and real) stock returns and inflation has been extensively analyzed in the literature, and many of papers have found a negative correlation between real stock returns and inflation, rejecting, the Fisher and the Wealth Effect hypotheses (Barsky, 1987; Bodie, 1976; Fama, 1981; Fama and Schwert, 1977; Kaul and Seyhum, 1990; Koustas and Serletis, 1999; Lintner, 1975; Ghazali and Ramlee, 2003; Koustas and Lamarche, 2010; Tsong and Lee, 2013). Thus, several theories alternatives to the Fisher Hypothesis have emerged in the literature to try to explain the negative correlation between real stock returns and inflation. For example, Modigliani and Cohn (1979) hypothesize that stock market investors fail to understand inflation's effect on the nominal cash flow, and in the presence of sustained inflation the valuation errors will induce an undervaluation of stocks - the Money Illusion Hypothesis. Feldstein (1980) argues that sustained increases in inflation reduce real stock prices since the artificial capital gains due to inflation will increase corporate tax liabilities and will reduce real after-tax earnings- the Tax Effect Hypothesis. Fama (1981) shows that the negative relationship between real stock returns and expected inflation is generated by a positive relationship between the stock market and economic activity and the negative correlation between inflation and real activity- the Proxy Effect Hypothesis. Geske and Roll (1983) suggest that stock price's reaction is in anticipation of future economic activity and is highly correlated to government revenues. When economic output decreases and the government faces a deficit, the Treasury either borrows or issues money, causing inflation. Thus, stock returns and inflation are negatively related due to a fiscal and monetary linkage - the Reverse Causality Hypothesis-. It is clear that, there are many theories which try to explain the relationship between stock returns and inflation rates, with very different empirical implications. Policy implications will also be very different depending on the theory used to explain the connection between these two variables.

Empirical evidence on these hypothesis has been mixed, although most empirical studies that reject the Fisher hypothesis have examined the relationship between inflation and stock returns at shorter horizons (Lintner, 1975; Bodie, 1976; Fama and Schwert, 1977; Fama, 1981; Gertler and Grinols, 1982; Kaul, 1987; Kaul and Seyhum, 1990; Hess and Lee, 1999), while, as the horizon increases, the results are more likely to support the Fisher hypothesis (Boudoukh and Richardson, 1993; Solnik and Solnik, 1997; Schotman and Schweitzer, 2000; Lothian and McCarthy, 2001). Lothian and McCarthy (2001), for example, find that equities are an inflation hedge, although this is only the case over very long periods. Cagan (1974) also finds that equity markets did adjust to inflation, but that the adjustment period lasted more than a decade. Two empirical results found in this literature are worth mentioning. First, some papers show that the sign and strength of the correlation between stock returns and inflation depend on the frequency scale. For example, Kim and In (2005), applying a wavelet analysis, show that there is a positive relationship between stock returns and inflation at the shortest scale (1-month period) and at the longest scale (128-month period), while a negative relationship is shown at the intermediate scales. Arouri et al. (2014) test for the Fisher hypothesis using data for Pakistan through a wavelets analysis and find that stocks could be used as a hedge against inflation in the long-run. Second, the results reveal that correlations between inflation and stock market returns are evolving heterogeneously overtime (Valcarcel, 2012; Antonakakis, Gupta and Tiwari, 2017). In this paper, we allow for these two possibilities (different correlation levels between inflation rates and stock market returns at different frequency scales and at different moments in time) by means of using different wavelets transforms for testing whether stock market returns can be considered an inflation hedge in a sample of two developed and two developing countries.

Fan and Gençay (2010) documented that the wavelets are helpful because of its appealing nature to deal with frequency components that are non-stationary such that they may appear, disappear, and then reappear over time, and traditional spectral tools may not account for such frequency components. They further added that the wavelet filters provide a natural platform to deal with the time-varying characteristics found in most real-world time series (most economic/financial time series exhibit quite complicated patterns over time (e.g. trends, abrupt changes, and volatility clustering)), and where the assumption of stationarity is violated. One can categorize wavelets into three main types such as: discrete, continuous and fast wavelets. Literature in the area of economics and finance is mostly concentrated with application of several variants of the discrete wavelet transform (DWT) due to the simplicity of the DWT and the advantages for data decomposition of many variables at the same time. Recently, the tools associated with the continuous wavelet transform (CWT) have become popular in addressing the same question. Though, Gençay, Selçuk, and Whitcher (2002) documented that the CWT is computationally complex and contains a high amount of redundant information, but it is also rather finely detailed. However, we argue that as compared to the DWT, the CWT approach offers a better interpretation of the variance, at different timescales. The relationship in time series data between two variables, what one may get by utilizing any method of DWT at each scale, can be obtained more easily with CWT without relying on any other econometric techniques. Specifically, with the CWT, the variation in the time series data can be obtained more easily and with a single diagram, one can immediately conclude the evolution of the variable variances at several time scales. Besides, the identification of common features in the time series variables is simplified through a single diagram and which is helpful in identify regions in the time-frequency space where co-movement is high and regions where co-movement is low and therefore, where phase differ- 
ences are meaningful. As documented in Bhanja et al. (2012), the CWT helps to unravel local correlation and local causal relationship over different time periods and frequencies without relying on traditional econometric techniques.

In this context, the objective of this paper is to test whether stock returns can be considered an inflation hedge for the UK economy, using a long span of data which covers observations for over the last three centuries (February 1790-February 2017). Furthermore, we compare the results obtained for the UK with those for the US and two developing countries (India, South Africa). The main contributions of the paper are the following. First, although our primary interest is to analyze the relationship between inflation rates and stock returns in the UK, our sample of countries in the empirical analysis includes two developed countries (the UK and the US) and two emerging countries (India and South Africa). Inflation rates in emerging countries have been higher and more volatile than in developed countries (Mitchell 1998) and have also been characterized by significant changes over time, including the recent inflation episode due to the commodity price increase in 2007-2008, which mostly affected emerging economies. Furthermore, financial markets in developing countries are less developed and investors have a narrower range of other inflation hedges to choose from than do investors in developed countries. Since all these factors could affect the stock-returns-inflation relationship, we believe that comparing the case of the UK economy with those of two developing countries (India and South Africa) adds an additional importance to our analysis. Second, the analysis covers the time period from February 1790 to February 2017, a period of time in which both stock returns and inflation rates have evolved heterogeneously over time, which suggests that the strength and sign of the relationship between the two variables are likely to have changed over the analyzed period. The nature of this long period makes more appropriate the use of the methodology employed in the paper. Finally, our main contribution is to examine the relationship between these two variables by means of a wavelet coherency analysis in the time and frequency domains. Wavelet coherency and phase differences simultaneously evaluate how causalities between stock returns and inflation rates fluctuate across frequencies and vary over time. This allows us to obtain short-term (high frequency) and long-term (long-frequency) relationships between the two series, and thus controls for potential nonlinearities and structural breaks in the relationship between the variables. The same methodology was used by Bhanja et al. (2012) to analyze the relationship between these variables in the Indian case, although, to the best of our knowledge, our paper is the first one that uses a wavelet approach to explore the relationship between the stock returns and inflation rates for the UK economy, the US, India and South Africa using over three centuries of data.

Our main results suggest that inflation rates and nominal stock returns present common movements, especially in the medium- and long-run. The results also confirm that the relationship between the two variables has changed over time during the long sample historical time period considered. Overall, our results tend to suggest that, while the relationship between stock returns and inflation rates varies across frequencies and time periods, there is no evidence of stock prices acting as an inflation hedge, irrespective of whether we look at the two developed or the two developing markets in our sample.

The remainder of the paper is structured as follows. Section 2 describes the methodology. Section 3 presents the data and the main empirical results, while Section 4 contains some concluding comments and policy implications.

\section{Methodology and data}

\subsection{Methodological aspects}

Through wavelets, the correlation levels are assessed at different frequency scales and at different moments in time. Practically, each time series is decomposed in different frequencies and this decomposition can be made using different wavelets transforms (i.e. discrete, continuous, multiple). Our paper relies particularly on the CWT which provides time-frequency evolution of a series under consideration and across all the times and frequencies the coherence structure between stock returns and inflation. This section provides only elementary notions about the CWT (for a detailed description see Torrence and Compo, 1998; Grinsted, Moore and Jevrejeva, 2004; Aguiar-Conraria, Azevedo and Soares, 2008; Rua and Nunes, 2009; Tiwari, 2013).

The wavelet transform decomposes a time series in functions (wavelets) localized both in time and frequency $\psi_{\tau, s}(t)$. For a discrete time series $x(t)$, the CWT is:

$$
W_{x}(\tau, s)=\frac{1}{\sqrt{s}} \sum_{t=1}^{N} x(t) \psi^{*}\left(\frac{t-\tau}{s}\right)
$$


Torrence and Compo (1998) show that for a discrete time series, the CWT is defined as a convolution and can be efficiently computed as a product in the Fourier space where the Morlet wavelet (with $\omega_{0}=6$ ) is a good choice in decomposing a signal.

Consequently, the studies using the wavelet coherence for assessing the co-movements of financial series usually resort to the Morlet wavelet, defined as:

$$
\psi(t)=\pi^{-\frac{1}{4}} e^{i \omega_{0} t} e^{-\frac{1}{2} t^{2}}
$$

where: $\omega_{0}$ is the frequency dimension and $t$ is the time dimension.

The corresponding Fourier transform is given by:

$$
\hat{\psi}(\omega)=\pi^{\frac{1}{4}} \sqrt{2 e}^{-\frac{1}{2}\left(\omega-\omega_{0}\right)^{2}} .
$$

The wavelet power spectrum (WPS), which shows the variance of the time series (i.e. signals) across time-scale, is defined by $\left|W_{x}(\tau, s)\right|^{2}$, while the white-noise and red-noise wavelet power spectra is (Torrence and Compo, 1998): ${ }^{2}$

$$
D\left(\frac{\left|W_{x}(\tau, s)\right|^{2}}{\sigma_{x}^{2}}<p\right)=\frac{1}{2} P_{k} \chi_{\nu}^{2}(p)
$$

where: $v$ is equal to 1 for real and 2 for complex wavelets; $P_{k}$ is the mean spectrum at the Fourier frequency $k$.

In addition, the wavelet coherence (WTC) method allows the estimation of the presence of a simple causeeffect relationship between the phenomena recorded in the time series. Torrence and Webster (1999) define the WTC of two time series with $W_{x}(\tau, s)$ and $W_{y}(\tau, s)$ wavelet transforms, as the absolute value squared of the smoothed cross-wavelet spectrum, normalized by the smoothed wavelet power spectra:

$$
R^{2}(\tau, s)=\frac{\left|S\left(s^{-1} W_{x y}(\tau, s)\right)\right|^{2}}{S\left(s^{-1}\left|W_{x}(\tau, s)\right|^{2}\right) \cdot S\left(s^{-1}\left|W_{y}(\tau, s)\right|^{2}\right)}
$$

where: $S(\cdot)$ is a smoothing operator and $s$ is the wavelet scale.

However, the CWT suffers from edge effects due to the fact that wavelets are not completely localized in time. Thus, to address this issue, we use the cone of influence (COI). Outside the COI, the edge effects are predominant and can distort the result. Further, the phase relationship is computed using the circular mean of the phase over regions with greater than 5\% statistical significance that are outside the COI. The circular mean of a set of angles $\left(a_{t}, t=1, \ldots, n\right)$ is defined as follows:

$$
a_{m}=\arg (A, B)
$$

where: $A=\sum_{t=1}^{n} \cos \left(a_{t}\right)$ and $B=\sum_{t=1}^{n} \sin \left(a_{t}\right)$.

Further we define the phase difference as follows, which shows any lag or lead relationships between components,

$$
\phi_{x, y}=\tan ^{-1} \frac{I\left\{W_{n}^{x y}\right\}}{R\left\{W_{n}^{x y}\right\}}, \phi_{x, y} \in[-\pi, \pi]
$$

where, $I$ and $R$ are the imaginary and real parts, respectively, of the smooth power spectrum. A phase difference of zero indicates that the time series move together (analogous to positive covariance) at the specified frequency; if $\phi_{x, y} \in[0, \pi / 2]$, the series move in-phase, with the time-series $y$ leading $x$; if $\phi_{x, y} \in[-\pi / 2,0]$, the series move in-phase, with the time-series $x$ leading $y$. We have an anti-phase relation if we have a phase difference of $\pi$ (or $-\pi$ ). If $\phi_{x, y} \in[\pi / 2, \pi]$, there is anti-phase relation with $x$ leading $y$ and if $\phi_{x, y} \in[-\pi,-\pi / 2]$, there is anti-phase relation with $y$ leading $x$. 


\subsection{Data}

Our data consists of monthly nominal and real stock returns and inflation for the UK - our main focus, and then also for two emerging markets (India and South Africa), and a developed market (US). The choice of these countries is based on availability of historical data, and provides a comparison of the results for UK with that of emerging and developed economies. Nominal stock prices are derived from Global Financial Database, and are converted to nominal stock returns by using the first differences of natural logarithms expressed in percentages by multiplying with 100; i.e. we consider log returns. For UK, we use the wholesale/producer price index as a measure of the price level, which in turn, is derived from the Three Centuries of Data (Version 2.3) maintained by the Bank of England at: http://www.bankofengland.co.uk/research/Pages/datasets/default.aspx. This data is available till April, 2016. The data is then updated until the end of our sample period from International Financial Statistics of the International Monetary Fund. We use this measure of price level for the UK rather than Consumer Price Index (CPI), since CPI data is only available from $1914 .^{3}$ For the remaining three countries used in comparison, we use the CPI as a measure of price level. The CPI data for the India and South Africa are derived from the Global Financial Database. For the US, the data is obtained from the data segment of Professor Robert J. Shiller's website: http://www.econ.yale.edu/ shiller/data.htm. Inflation is computed as month on month changes in the natural logarithms of the measure of price level expressed in percentages. Real stock return is then derived by subtracting the inflation rate from the nominal stock returns. Based on data availability the sample of data covered are: 1790:02-2017:02 for UK; 1920:08-2017:02 for India; 1936:01-2017:01 for South Africa; and 1871:02-2017:02 for US. The data on nominal and real stock returns, and inflation have been plotted in Figure 4 in the Appendix of the paper. The online version of this article offers supplementary material (DOI: https://doi.org/10.1515/snde-2017-0049).

\subsection{Descriptive statistics, correlations and wavelet energy distribution}

The descriptive statistics of the nominal and real stock returns and inflation rates, together with the correlation coefficients between nominal and real stock returns and inflation rates, and the normality and white noise properties of the series are presented in Table 1.

Table 1 is divided in three sections. The first section (at the top) shows the descriptive statistics, while the second (at the middle) and the third (at the bottom) sections present the correlations structure and the normality and white noise properties of the data series. The descriptive statistics suggest that the lowest median nominal and real stock returns correspond to India and the highest to the UK, while the highest inflation rates also correspond to India. Furthermore, the variance of the series takes the highest value in the case of India, suggesting that the volatility of the series in this country is higher than in the others. The second section of the table provides evidence that correlation coefficients between nominal stock returns and inflation rates are positive but low, while the correlation coefficients between real stock returns and inflation rates are negative and relatively larger. The third section of the table presents the normality results (conducted through Jarque-Bera test), which reveals that stock returns and inflation series in each country depart significantly from the normal distribution. The Ljung-Box Q-statistic and Box-Pierce test statistic at lag 6 and 12 show evidence of significant serial dependence in nominal and real returns and inflation series in each country. The McLeod-Li test statistics provides evidence of ARCH effects in all series in all countries (with the exception of India for nominal and real stock returns). 


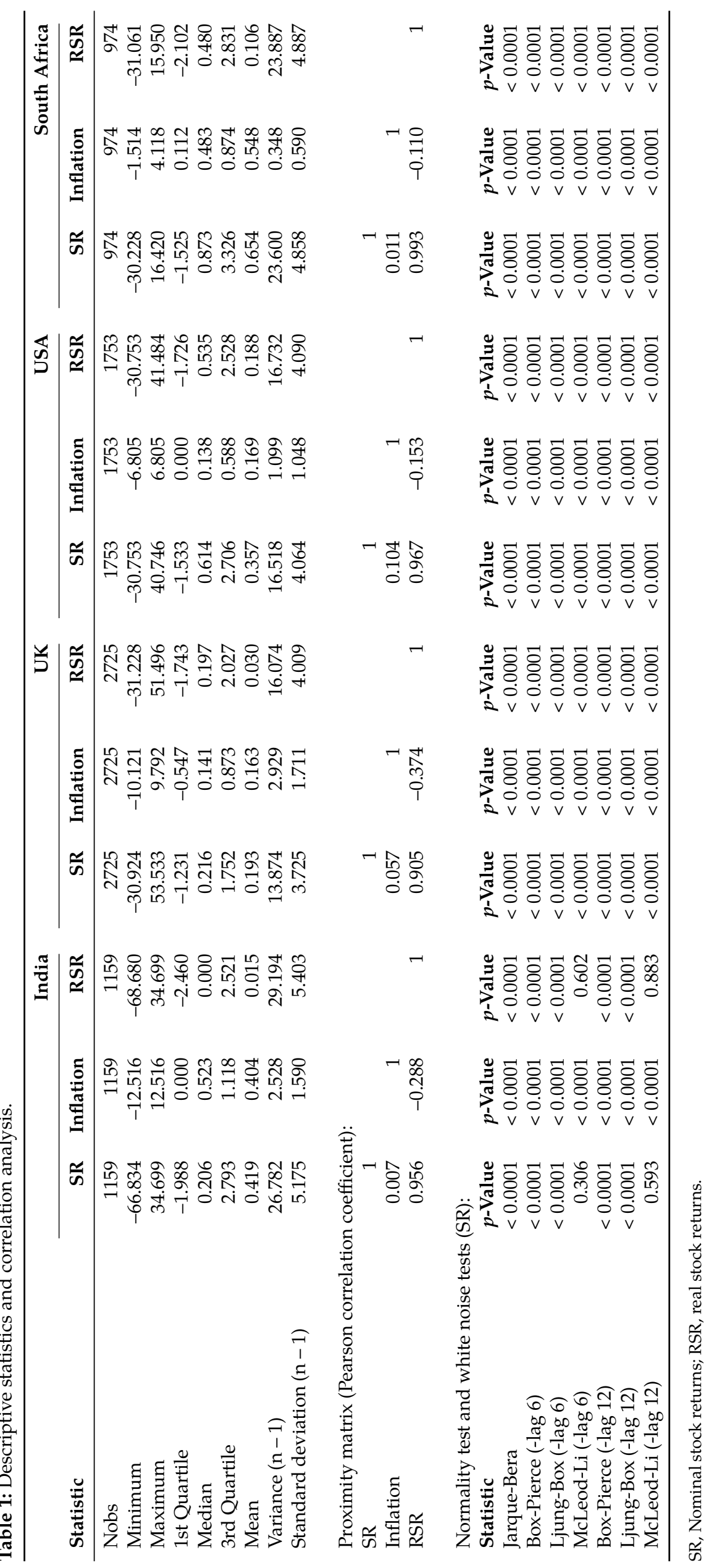


Table 2 presents the "wavelet energy decomposition" of each of the series for each country. These decompositions are obtained by using the LA8 wavelet filter and decomposing the series up to level 8 . Note that the reported values represent the percentages of the signal energy (variance) at various frequency intervals. The wavelet details D1-D8 correspond to 2-4, 4-8, 8-16, 16-32, 32-64, 64-128, 128-256, and 256-512 monthly cycle variations, whilst the $\mathrm{S} 8$ component reveals oscillations beyond 512 monthly cycles.

Table 2: Wavelet energy decomposition of SR, RSR and inflation.

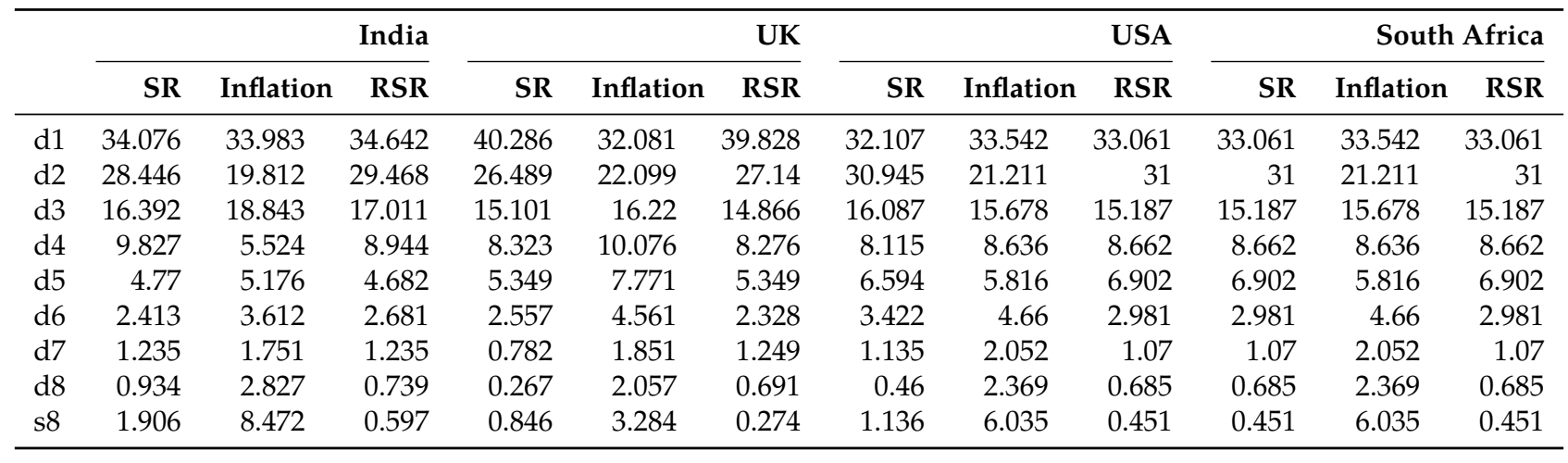

The reported values are percentages.

The energy distribution shown in Table 2 can be grouped in four periods, namely short-run (D1+D2), medium-run (D3+D4+D5+D6), long-run (D7+D8), and very long-run (S8) periods of fluctuations. It is evident from Table 2 that the maximum energy distribution for each of the series is found in the short run (an observation, we also confirm below more formally over time), while in the very long run the energy distribution is about 10 percent.

\section{Empirical findings}

The results for each country under consideration is presented in Figure 1-Figure 3 through the wavelet power spectrum (WPS), and its average power spectrum, cross-wavelet transform (XWT) and its average power spectrum, wavelet coherency (WC) and phase-differences. The output of WPS, XWT and WC is illustrated by contour plots arranged in three dimensions: frequency and time spaces (i.e. the vertical and horizontal axes of the contour plots), and the time-scale wavelet spectrum values (i.e. the colored spectrum of increasing intensity from dark blue to red). More specifically, an increasing value of the wavelet spectrum matches up with a deepening red color. The frequency is converted into time units (months), and it ranges from the highest frequency of 2 months (top of the plots) to the lowest frequency of 512 months (bottom of the plots). The time on the horizontal axis indicates the whole sample period for each country under consideration. A significant red color at the extreme left/right corner indicates the existence of extreme events at the beginning/end of the period, while the same color concentration at the bottom/top implies the occurrence of significant scenarios at low/high frequencies. A visual assessment of the figures shows that both extreme time and frequency movements are detected. 

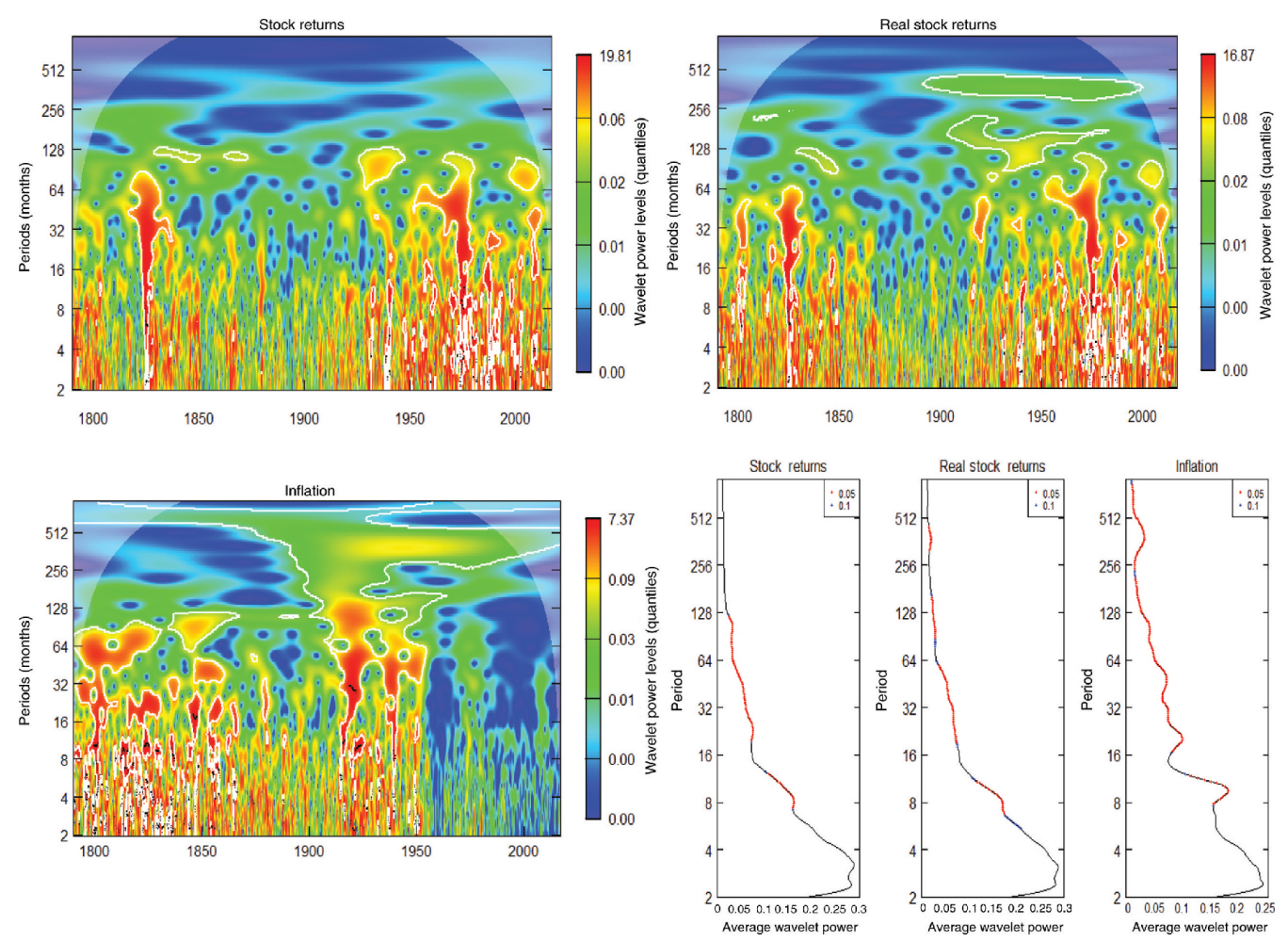

Figure 1: WPS and average wavelet power for UK.
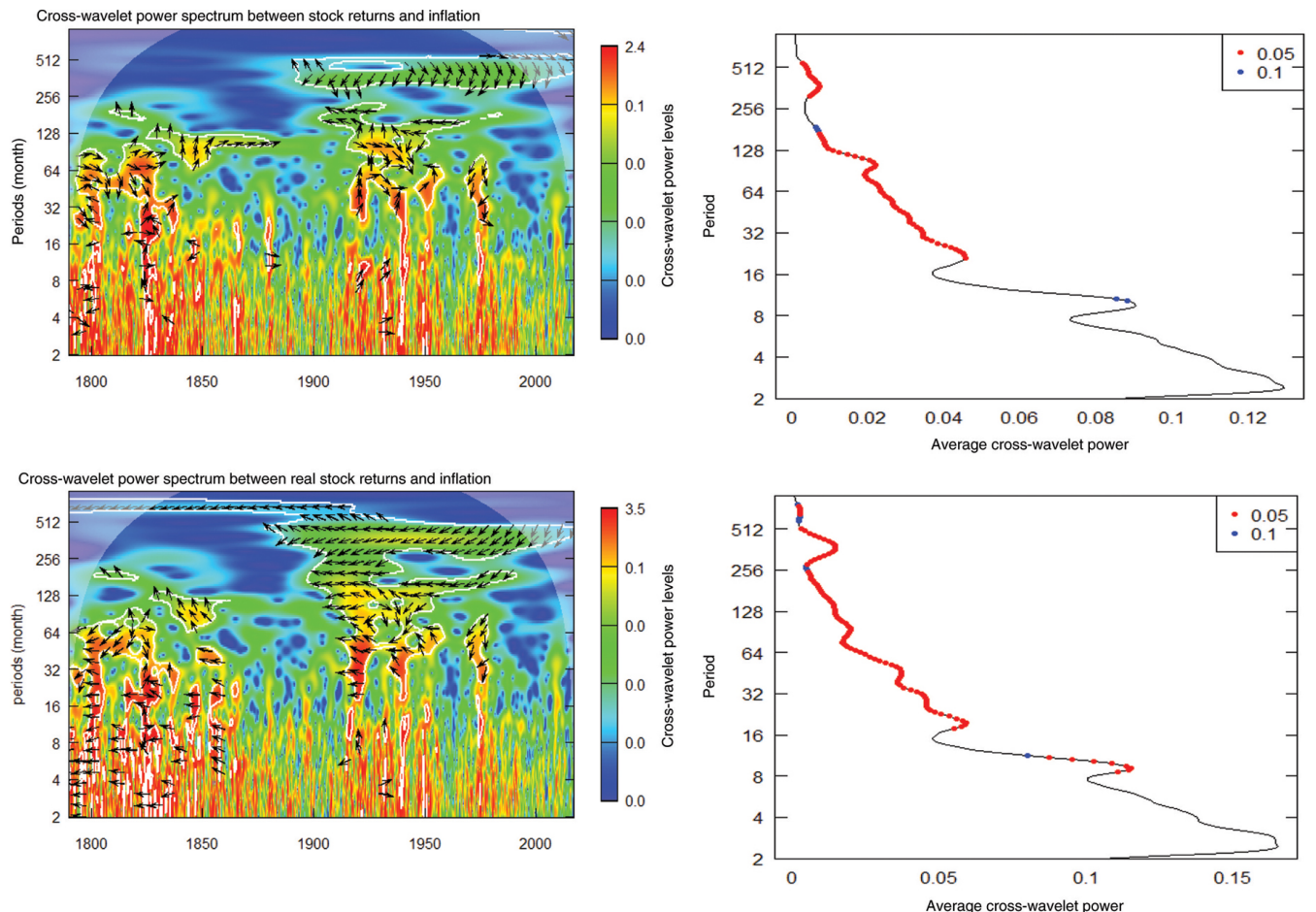

Figure 2: Cross-wavelet transform (XWT) and average wavelet power for UK. 

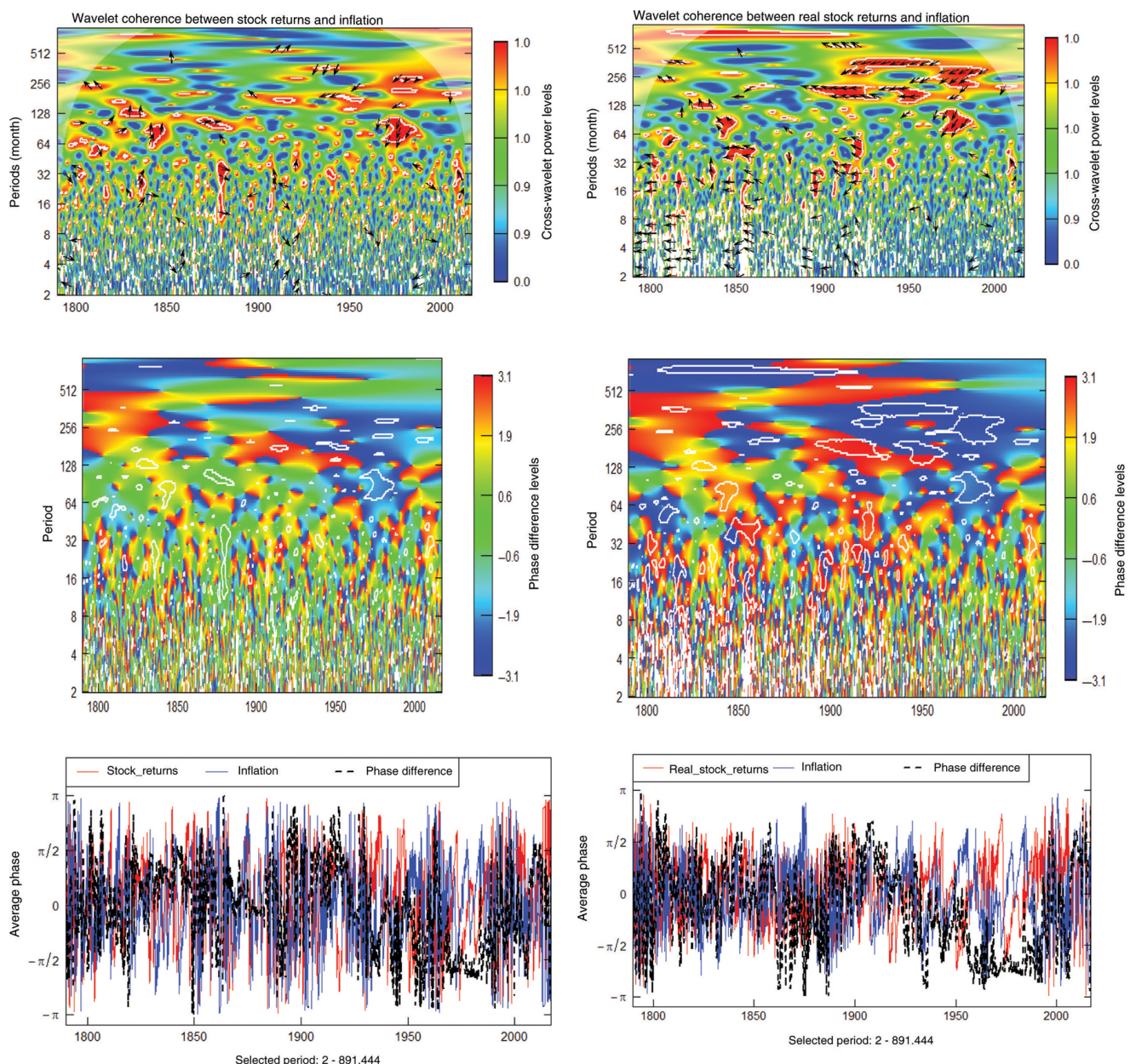

Figure 3: Continuous wavelet coherency (CWT) and phase-differences for UK.

\subsection{Wavelet analysis for UK}

Since the focus of our paper is the UK, we present the results for UK first and in great details. The WPS (which is sometimes referred as Local Wavelet Power Spectrum (LWPS) in the literature) results for UK is presented in Figure 1 in four parts. In the left-upper corner we present WPS of nominal stock returns and right-upper corner we present results of WPS of real stock returns. Left-lower corner presents the WPS of inflation and right-lower corner presents average wavelet power (also known as Global Wavelet Power Spectrum (GWPS) which gives the averaged variance contained in all wavelet coefficients of the same frequency) of nominal stock returns, real stock returns and inflation, respectively. The WPS is helpful in identifying the similarities in the evolution of the data series across time-scale. Without being a direct proof of either co-movements or lead-lag relationships, the results of the WPS can be interpreted as a first sign of interdependences between nominal and real stock returns and inflation.

It is evident from Figure 1 that high wavelet power levels of nominal stock and real stock returns are in the same periods and frequencies. In particular, nominal stock and real stock returns showing high wavelet power levels between the 2 to 64 months scale, represented by red color contour, are around 1800, 1825, and since 1930 till 2016. However, for inflation, the period before 1950 represents high wavelet power in the 2-64 months scale. If we analyze the plots of average wavelet power, we find significant (at the $5 \%$ level) common months scale of 16-128 between nominal stock returns and inflation, and at the 16-230 months scale for real stock returns and inflation. To further validate these findings, we use the cross wavelet transform (XWT) and its average power spectrum.

It is very interesting to observe that in Figure 2, in general, the portrayed pattern of average cross-wavelet power is same for both nominal stock returns and inflation, and real stock returns and inflation. If we observe closely, we find that the average cross-wavelet power is consistently significant between the 16-512 months range, as well as between the 8-10 months scale, when real stock return is analysed with inflation. But for nominal stock returns, the average cross-wavelet power is significant between 16-128 months and between 256 and 512 months. In terms of the direction of arrows associated with the cross-wavelet power spectrum, they 
are not consistent and it is very difficult to draw conclusions, because for same period at different frequencies arrows are pointing right up or down, and left up or down. In other words, the direction of the arrows varies across various frequency bands over the time period under consideration. Even though it is difficult to draw clear-cut conclusions, one can observe a strong link between nominal and real stock returns with inflation as seen from the average cross-wavelet power figures. However, to get more insight about the lead-lag relationship, we now turn to wavelet-coherency and phase-differences, as presented in Figure 3.

While the WPS offers some insights about the probable co-movements of the series, the wavelet coherence, together with phase-difference, provides not only a good tool for a descriptive analysis of the relationship, but also for examining the co-movements and lead-lag relationships. We document that the variables under consideration depict common movements, especially in the medium- and long-runs. Figure 3 has two columns and three rows, with the first column presenting results for nominal stock returns and inflation, and the second column doing the same for real stock returns and inflation. The first row in Figure 3 depict results of waveletcoherence (and phase-differences through arrows), a global pattern of average phase difference is graphed in the second row to demonstrate phase-or anti-phase and lead and lag relationship between nominal or real stock returns and inflation, where the color code represents the power of phase difference, with it ranging from -3.1 (i.e. $-\pi$ ) (dark blue color) to 3.1 (i.e. $\pi$ ) (dark red color), and finally in the third row, averaged phases and phase-difference are plotted.

Our results based on wavelet-coherency (from the first row of the Figure 3) for both nominal stock returns and inflation, and real stock returns and inflation indicate arrows mostly pointing upward-left at the $4 \sim 64$ months scale over different periods. This implies that inflation and stock returns are out of phase (i.e. anticyclical) for most of the periods. Similarly, at more than 128 months scale, especially over the 1920-2017 period, inflation and stock returns are out of phase (negative relationship). In the second row of Figure 3, a global pattern of averaged phase difference is presented to demonstrate phase-or anti-phase and lead-lag relationship between stock returns and inflation. But to get a clearer understanding, we analyze the pattern of average phase differences presented in third row of Figure 3. We find that for the period prior to 1800, 1875-1890, and 19502000 , phase falls mostly between $[-\pi,-\pi / 2]$, indicating that there is anti-phase relation with inflation leading stock returns. The negative relationship between stock returns and inflation in turn can be due to the Money Illusion, Tax Effect, Proxy Effect, and/or Reverse Causality Hypotheses, and it has important economic and policy implications. For instance, and from the investors' point of view, this negative relationship would mean that investors would be better off in reducing their stock market investments in times of high inflation rates. Furthermore, this relationship would imply, for example, that tight monetary policies aimed to reduce inflation could not be used to reduce potential bubbles in stock markets. During the other periods (1800-1870, 19001950 and post 2000) inflation and stock returns are in phase $[-\pi / 2, \pi / 2]$, with stock returns leading inflation in general. In other words, evidence in favour of stock returns acting as an inflation hedge is virtually non-existent, even though, we do have episodes of positive relationship between stock returns and inflation, which in turn, are primarily due to the wealth effect.

\subsection{Comparison with the wavelet analyses for US, India and South Africa}

In this sub-section, we discuss the results obtained for the US - a developed economy, and that for two emerging markets of South Africa and India. In the process, we compare our results with that of UK presented above. As can be seen from Figure 5-Figure 13 in the Appendix of the paper, the relationship between inflation and nominal or real stock returns, especially at medium to long-run cannot be denied, as was also observed for the UK. Moreover, as with UK, the relationship is not only time-varying, but also contingent on the frequencies we are looking at. Most importantly, considering the average phase differences, we observe that in US and India, stock returns (nominal or real) are mostly anti-phase (i.e. negatively related) relative to inflation, with inflation leading stock returns, as in the UK. Whenever, in-phase movements are observed as in the early part of the sample for the US, and majority of the time-period for South Africa (barring 1970s and mid-1980s when the variables are anti-phase with inflation leading stock returns, and the period of 2000-2010 when the in the negative relationship between stock returns and inflation, the former is the lead variable), stock returns lead inflation, i.e. the wealth effect is at play. So, overall our results tend to suggest that, while the relationship between stock returns and inflation varies across frequencies and time periods, there is no evidence of stock returns acting as an inflation hedge, irrespective of whether we look at developed or developing markets based on long-samples of historical data. In other words, based on our study, we can conclude that stock returns are a bad hedge against inflation, and this result holds for the four countries analysed in this paper. Furthermore, the economic and policy implications suggested for the UK case in the previous section can be generalized to these countries, US, India and South Africa. 


\section{Conclusions}

The objective of this paper is to test whether stock returns can be considered an inflation hedge for the UK economy, using a long span of data which covers observations for over the last three centuries (February 1790February 2017), by means of applying different wavelets transforms (i.e. discrete, continuous, multiple). Using this methodology, we test for the generalized Fisher hypothesis allowing for different correlation levels between inflation rates and stock market returns at different frequency scales and at different moments in time. Furthermore, we compare the results obtained for the UK with those for the US and two developing countries (India, South Africa).

The main results of the paper can be summarized as follows. First, our main results suggest that the relationship between inflation rates and nominal stock returns changes at different frequencies and different time periods. In particular, both variables present common movements especially in the medium- and long-run. Second, in the long-run (at more than 128 months scale), and specially over the periods prior to 1800, 18751890 and 1950-2000, inflation and stock returns appear negatively related, with inflation rates leading stock returns. Based on the theories mentioned in the introduction, we could conclude that for these time periods, the Fisher and the Wealth Effect Hypothesis could be rejected in favor of the Money Illusion, Tax Effect, Proxy Effect and/or Reverse Causality Hypotheses. Several and relevant economic and policy implications can be derived from this result. First, and from the investors point of view, this negative relationship would mean that investors would be better off in reducing their stock market investments in times of high inflation rates. Furthermore, this relationship would imply, for example, that tight monetary policies aimed to reduce inflation could not be used to reduce potential bubbles in stock markets in periods where a negative relationship between stock returns and inflation rates are found. In contrast, and for the time periods 1800-1870, 1900-1950 and post 2000, characterized in general for lower inflation rates, the results suggest the existence of a positive relationship between the variables with stock returns leading inflation rates. That is, for these periods, the results support the Wealth Effect Hypothesis, but not the Fisher Hypothesis, so that we can conclude that stock returns are not a hedge against inflation. On the contrary, the results for these time periods suggest that increases in real stock returns will positively impact inflation through their effect on consumption and aggregate demand. Finally, when we compare the results for the UK case with those for the US, India and South Africa, they again suggest that there is no evidence of stock returns acting as an inflation hedge, irrespective of whether we look at developed or developing economies. The same policy implications can be derived for these countries as for the UK case. Again, investors in these countries could be better off reducing their investments in stock markets in periods of high inflation rates, while monetary authorities' policies could be less effecting in decreasing potential risks of stock market bubbles.

In general, monetary authorities and investors should analyze the sign and direction of the stock returns and inflation rates relationship before adopting any monetary policy or investment strategy. Finally, the investors and monetary authorities should also take into account that the relationship between the two variables is different at different frequencies. For example, investors should consider that their investment strategies will depend on their investment horizons and monetary authorizes should contemplate that monetary policies could have different effects on the short-run and the long-run.

\section{Acknowledgments}

Juncal Cunado gratefully acknowledges financial support from the Ministerio de Economia y Competitividad (ECO2017-83183-R). We would like to thank an anonymous referee for many helpful comments. However, any remaining errors are solely ours. 


\section{Appendix}
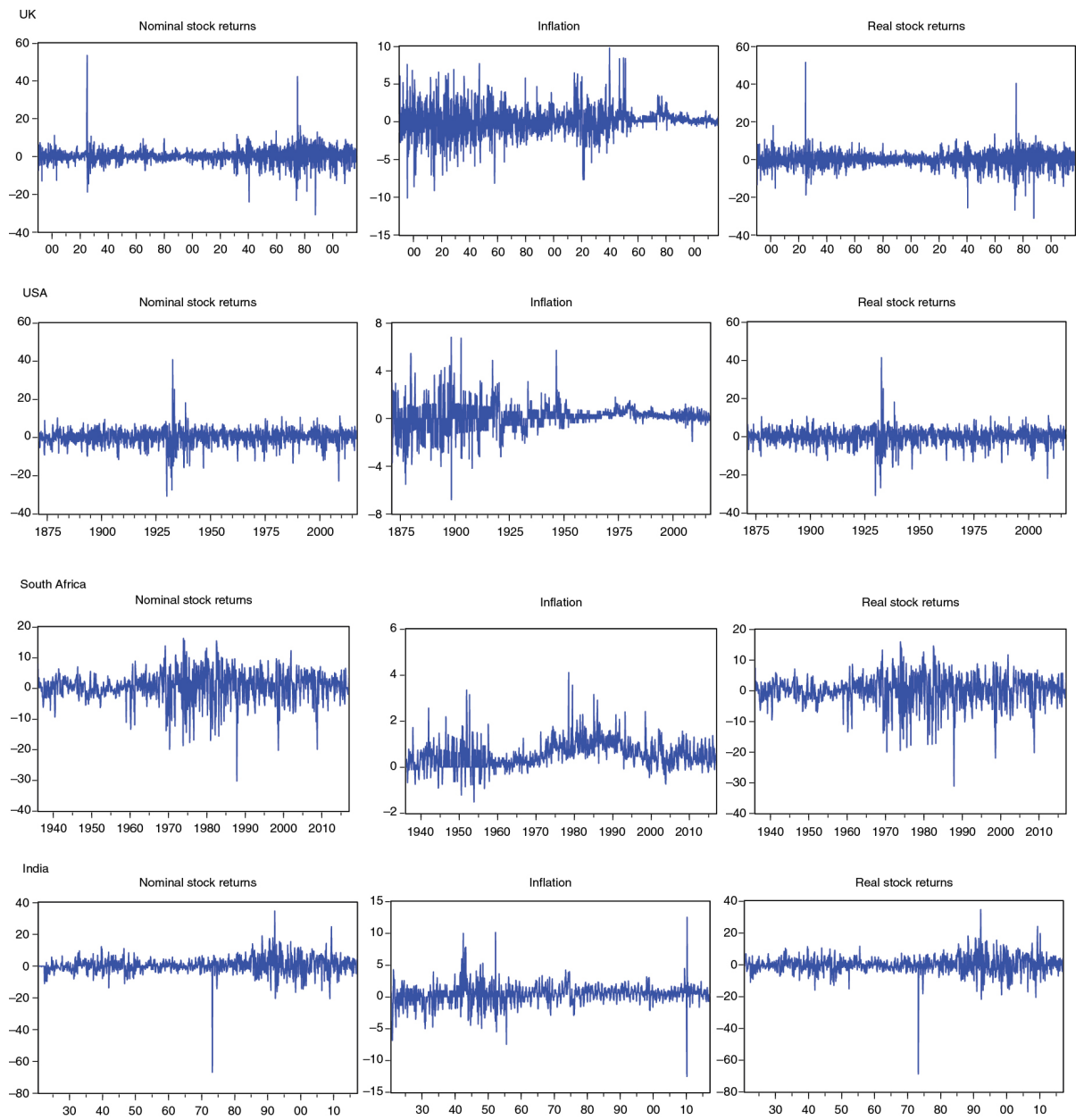

Figure 4: Nominal stock returns, inflation rates and real stock returns.
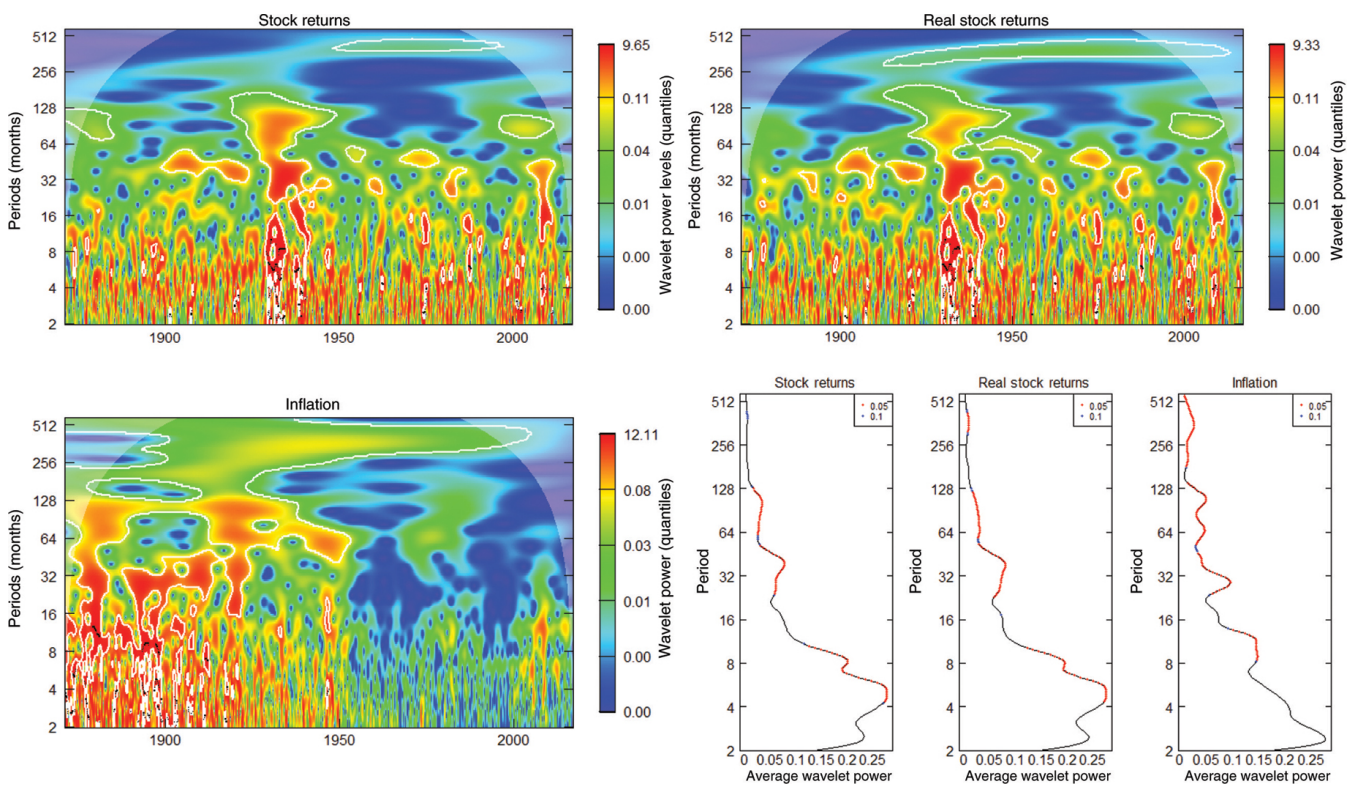

Figure 5: WPS and average wavelet power for US. 

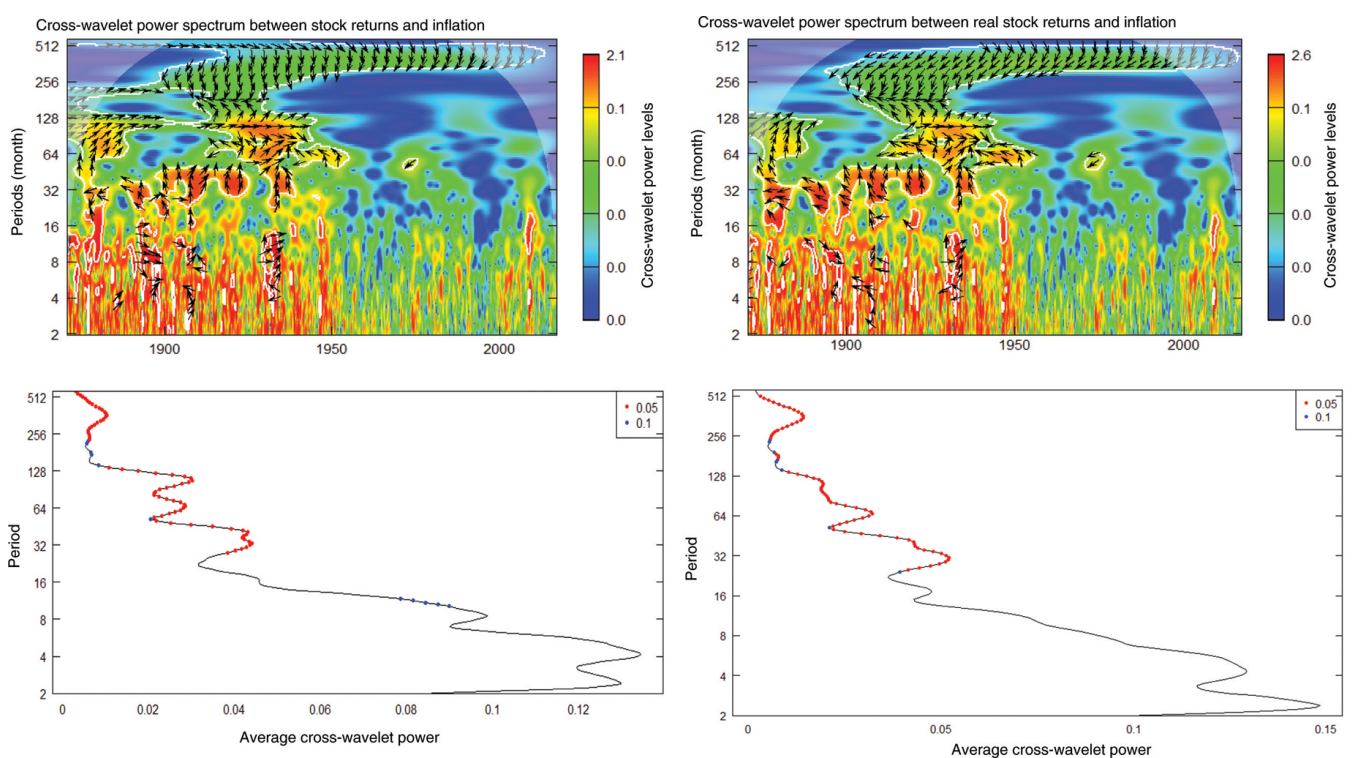

Figure 6: Cross-wavelet transform (XWT) and average wavelet power for US.
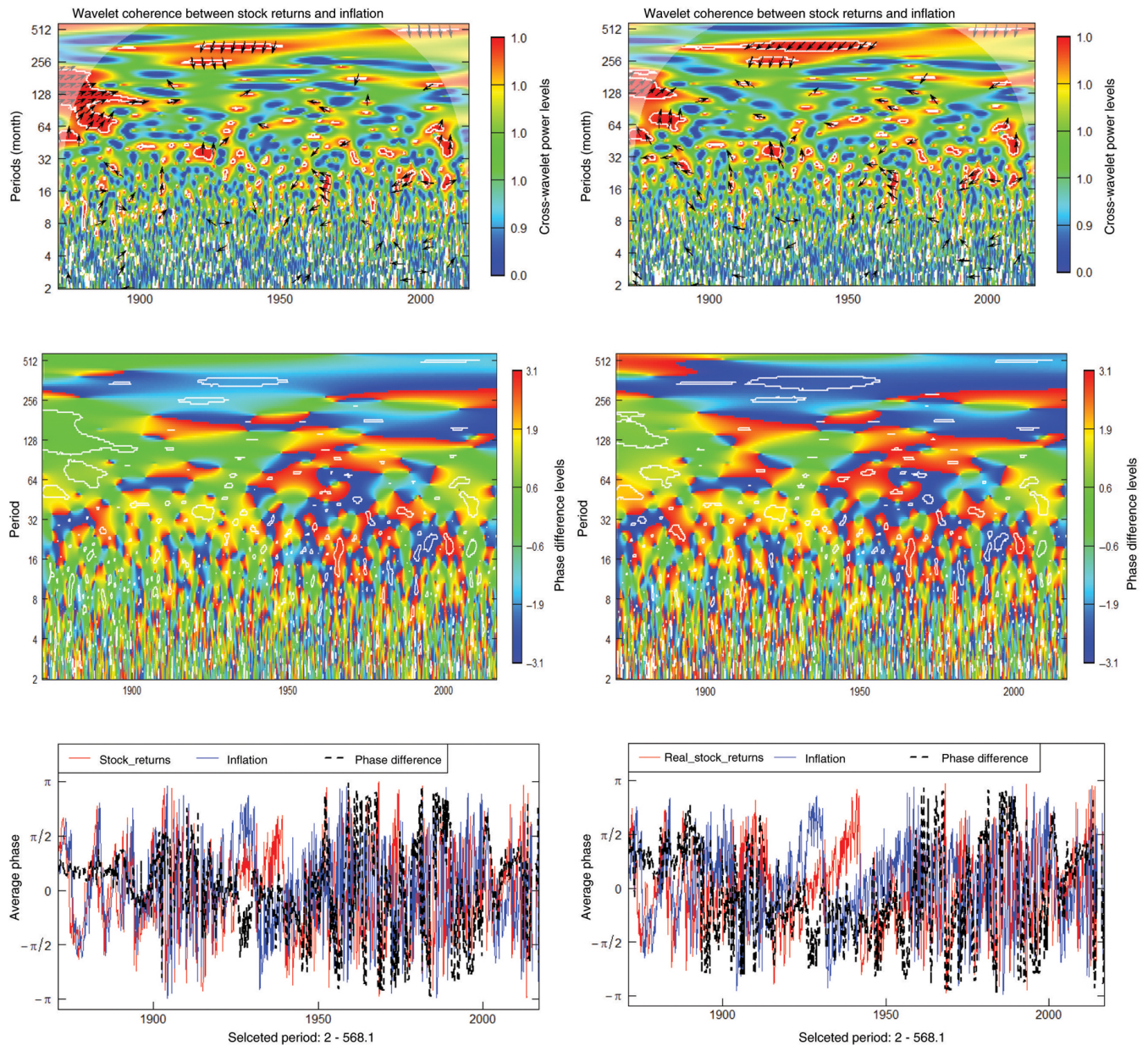

Figure 7: Continuous wavelet coherency (CWT) and phase-differences for US. 

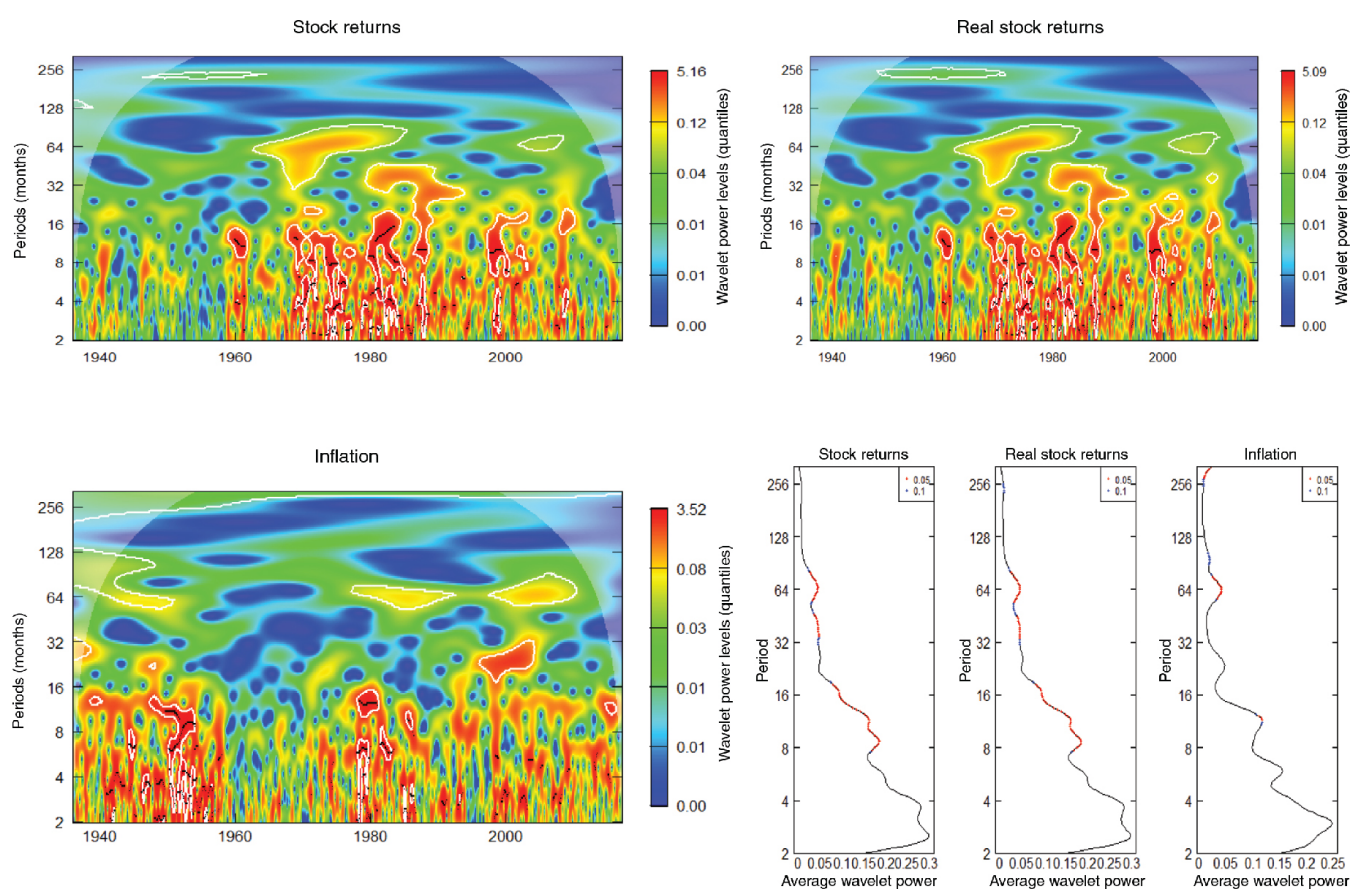

Figure 8: WPS and average wavelet power for South Africa.
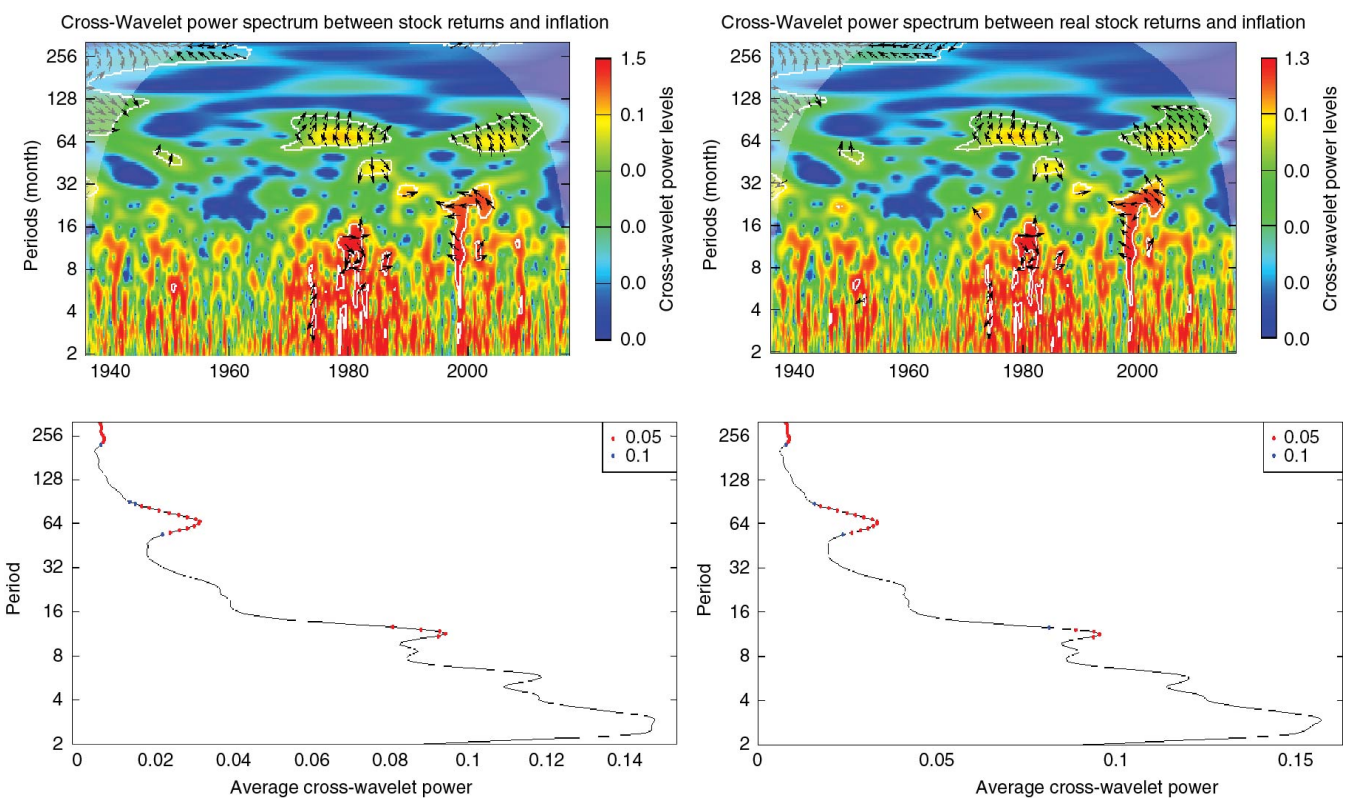

Figure 9: Cross-wavelet transform (XWT) and average wavelet power for South Africa. 

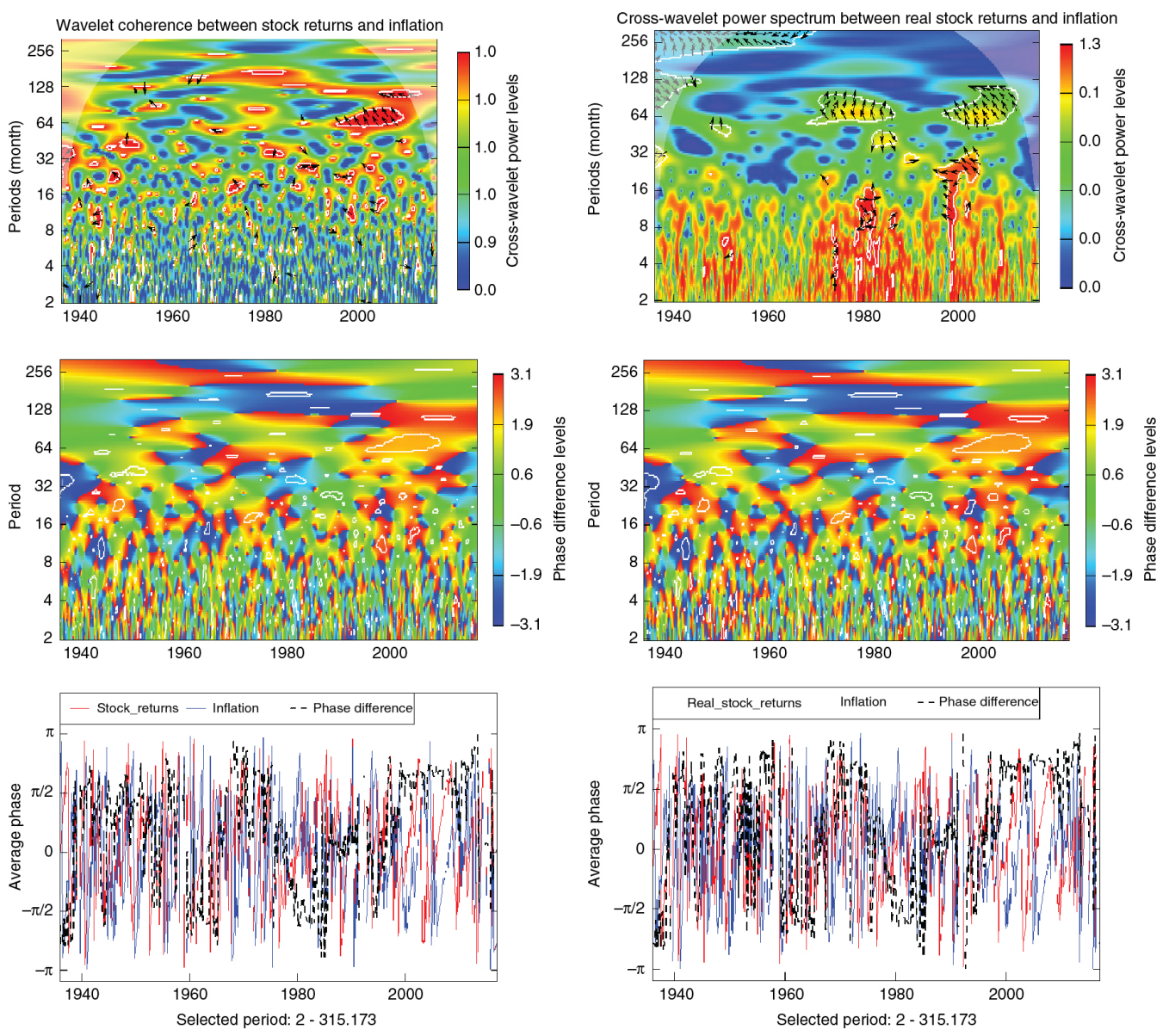

Figure 10: Continuous wavelet coherency (CWT) and phase-differences for South Africa.
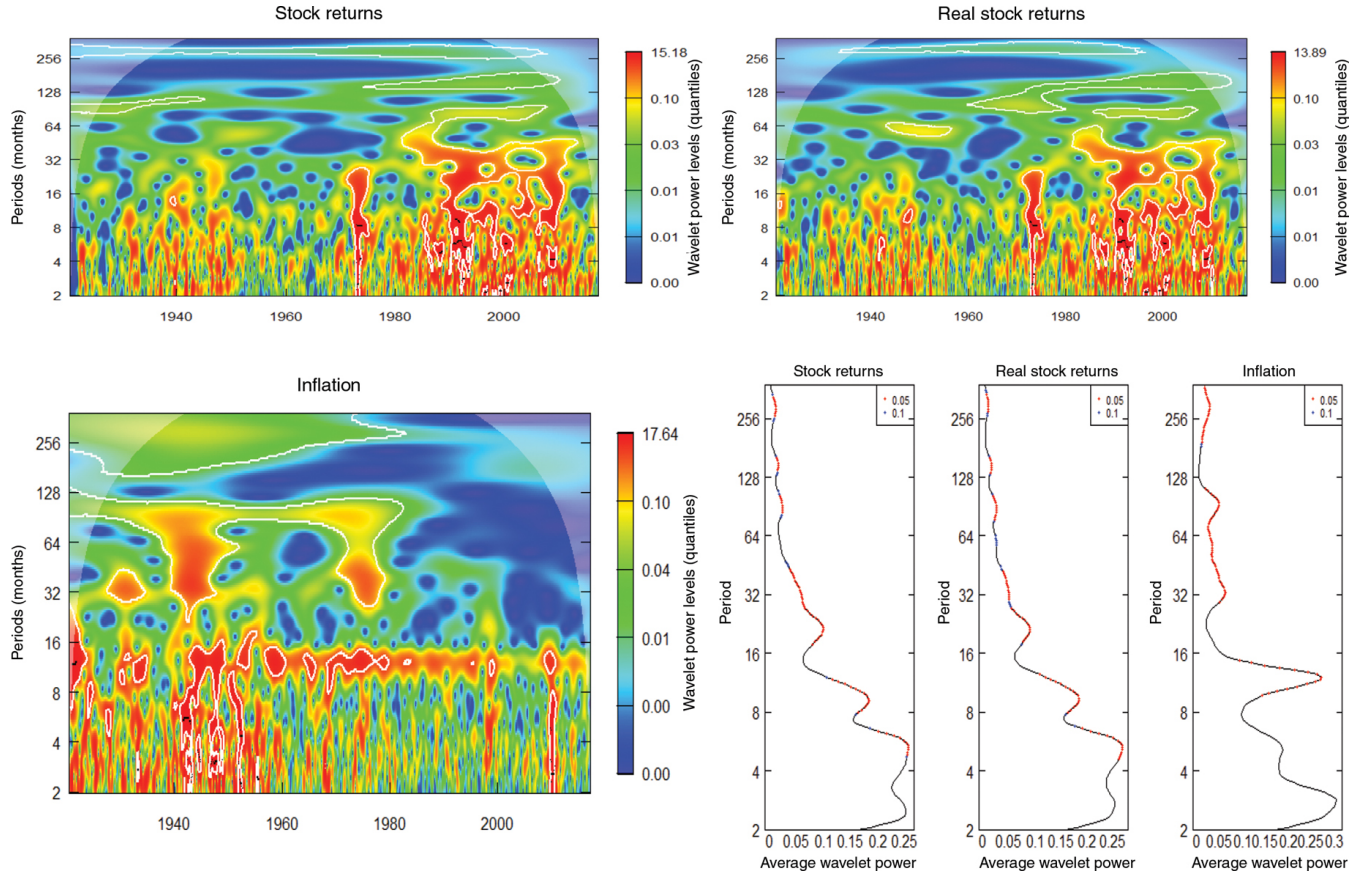

Figure 11: WPS and average wavelet power for India. 

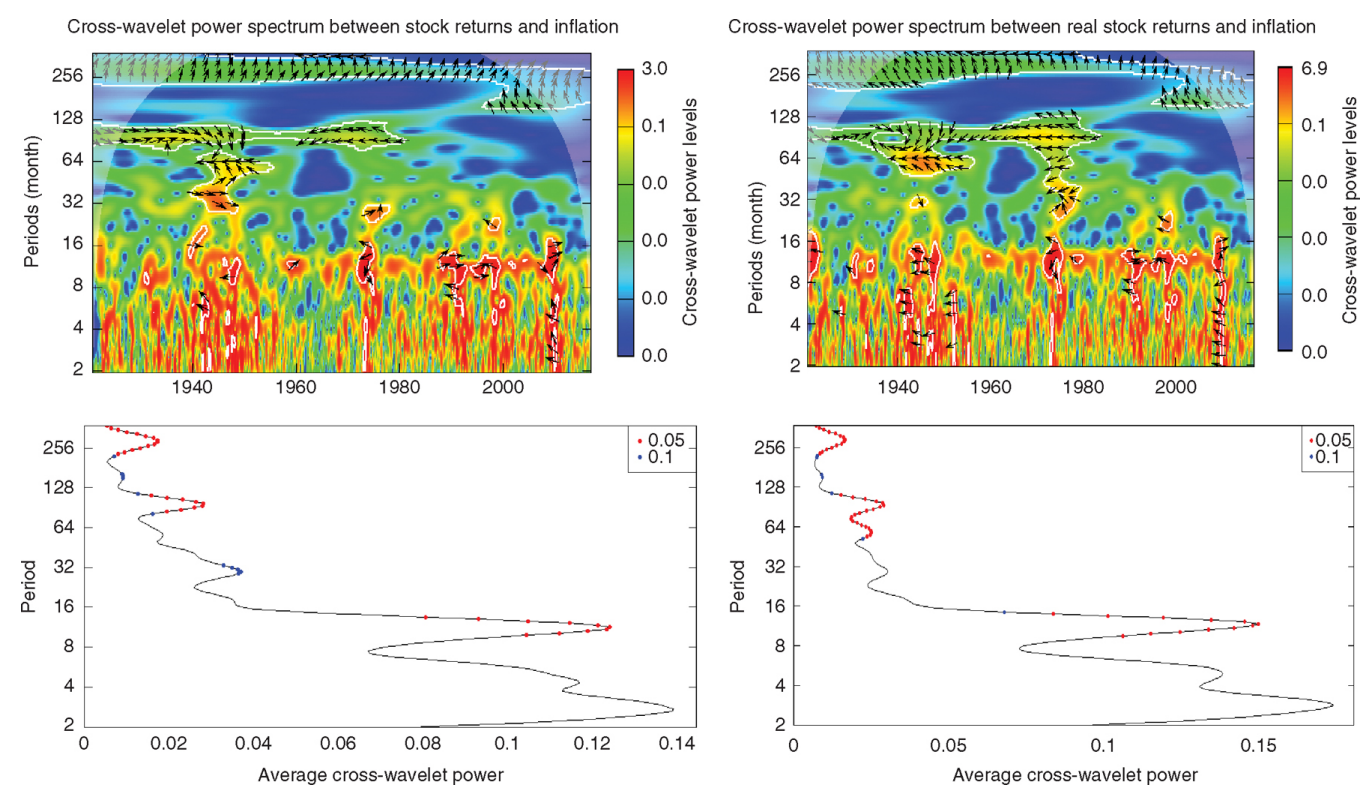

Figure 12: Cross-wavelet transform (XWT) and average wavelet power for India.
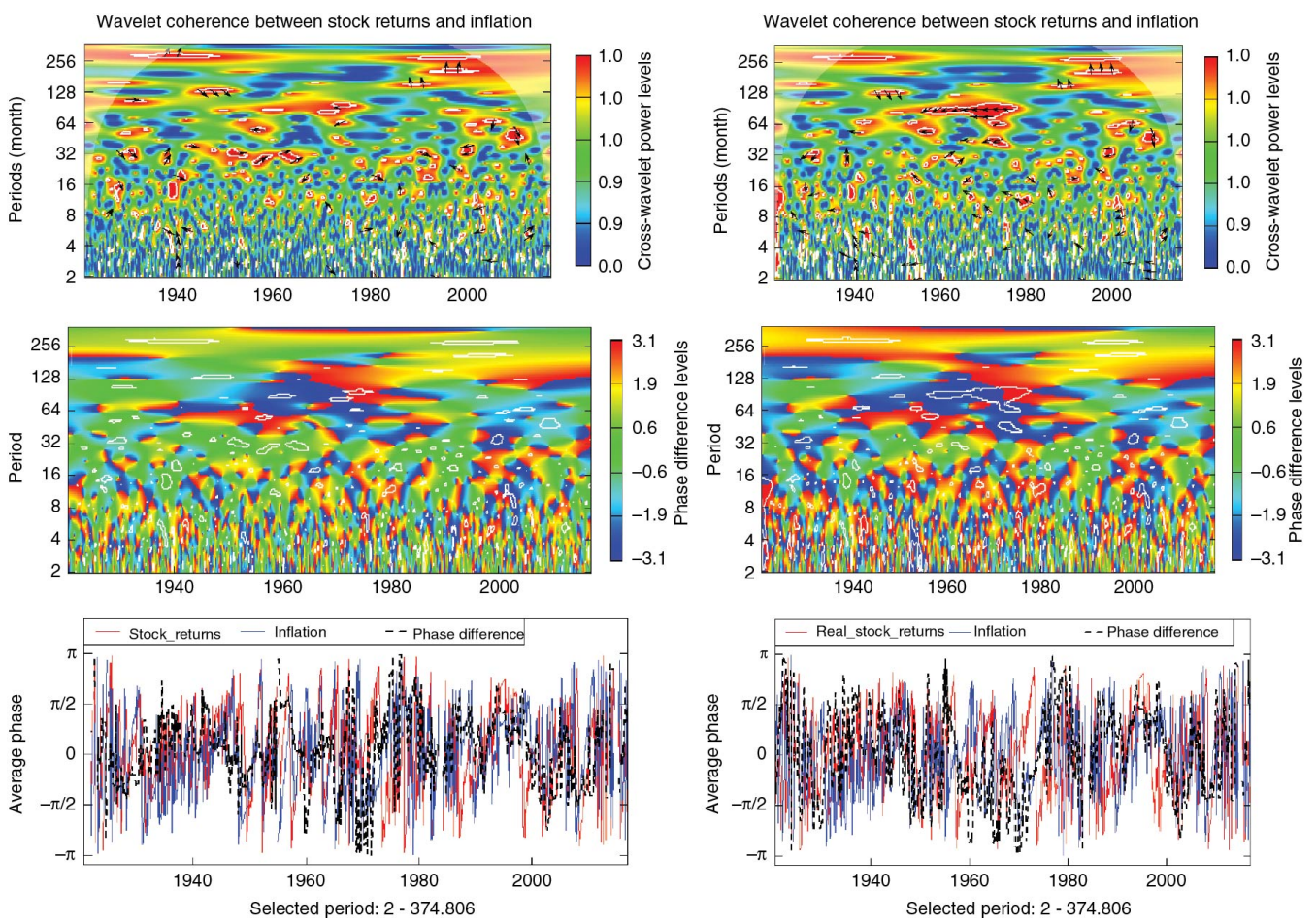

Figure 13: Continuous wavelet coherency (CWT) and phase-differences for India.

\section{Notes}

1 See O'Donoghue, Goulding, and Allen (2004) for a detailed description of inflation in the UK since 1750.

2 We use the theoretical distribution of the wavelet power spectrum for computing the significance levels.

3 Our results were however, qualitatively similar over the period of 1914:08-2017:02 if we used the CPI-based measure of inflation, rather

than the wholesale or producer price based inflation. Complete details of these results are available upon request from the authors.

\section{References}

Aguiar-Conraria, L., N. Azevedo, and M. J. Soares. 2008. "Using Wavelets to Decompose the Time-Frequency Effects of Monetary Policy." Physica A: Statistical Mechanics and its Applications 387: 2863-2878. 
Ando, A., and F. Modigliani. 1963. "The Life Cycle Hypothesis of Saving: Aggregate Implications and Tests." American Economic Review 53: 5584.

Antonakakis, N., R. Gupta, and A. K. Tiwari. 2017. "Has the Correlation of Inflation and Stock Prices Changed in the United States over the Last Two Centuries?" Research in International Business and Finance 42: 1-8.

Arouri, M., A. K. Tiwari, A. B. Dar, N. Bhanja, and F. Teulon. 2014. “Stock Returns and Inflation in Pakistan.” IPAG Business School, WP 2014108.

Barsky, R. B. 1987. “The Fisher Hypothesis and the Forecastability and Persistence of Inflation.” Journal of Monetary Economics 19: 3-24.

Bhanja, N., A. Billah, A. K. Tiwari, and O. R. Olayeni. 2012. "Are Stock Prices Hedge Against Inflation? A Revisit Over Time and Frequencies in India." Central European Journal of Economic Modelling and Econometrics 4: 199-213.

Bodie, Z. 1976. "Common Stocks as a Hedge Against Inflation." Journal of Finance 31: 459-470.

Boudoukh, J., and M. Richardson. 1993. “Stock Returns and Inflation: A Long-Horizon Perspective.” American Economic Review 83: 1346-1355.

Cagan, P. 1974. “Common Stock Values and Inflation: The Historical Record of Many Countries." National Bureau of Economic Research Annual Suplement, New York.

Fama, E. F. 1981. "Stock Returns, Real Activity, Inflation and Money." American Economic Review 71: 545-565.

Fama, E. F., and C. W. Schwert. 1977. “Asset Returns and Inflation." Journal of Financial Economics 5: 115-146.

Fan, Y., and R. Cençay. 2010. “Unit Root Tests with Wavelets.” Econometric Theory 26: 1305-1331.

Feldstein, M. 1980. "Inflation and the Stock Market." American Economic Review 70: 839-847.

Fisher, I. 1930. The Theory of Interest. New York: Macmillan.

Gençay, R., F. Selçuk, and B. Whitcher. 2002. An Introduction to Wavelets and other Filtering Methods in Finance and Economics. San Diego: Academic Press.

Certler, M., and E. L. Crinols. 1982. “Unemployment, Inflation, and Common Stock Returns.” Journal of Money, Credit and Banking 14: $216-233$.

Ceske, R., and R. Roll. 1983. "The Fiscal and Monetary Linkage Between Stock Returns and Inflation." Journal of Finance 38: 1-33.

Chazali, N. and S. Ramlee. 2003. “A long memory test of the long-run Fisher effect in the G7 countries." Applied Financial Economics 13: 763769.

Crinsted, A., J. C. Moore, and S. Jevrejeva. 2004. "Application of the Cross Wavelet Transform and Wavelet Coherence to Ceophysical Time Series." Nonlinear Processes Ceophysics 11: 561-566.

Hess, P., and B. D. Lee. 1999. "Stock Returns and Inflation with Supply and Demand Disturbances." The Review of Financial Studies 12: 12031218.

Kaul, G. 1987. “Stock Returns and Inflation: The Role of the Monetary Sector." Journal of Financial Economics 18: 253-276.

Kaul, G., and H. N. Seyhum. 1990. "Relative Price Variability, Real Shocks, and the Stock Market." Journal of Finance 45: 479-496.

Kim, S., and F. In. 2005. “The Relationship Between Stock Returns and Inflation: New Evidence from Wavelet Analysis." Journal of Empirical Finance 12: 435-444.

Koustas, Z., and A. Serletis. 1999. “On the Fisher Effect." Journal of Monetary Economics 44: 105-130.

Koustas, Z., and J. F. Lamarche. 2010. “Evidence of Non-Linear Mean Reversion in the Real Interest Rate." Applied Economics 42: $237-248$.

Lintner, J. 1975. “Inflation and Security Returns.” The Journal of Finance 30: 259-280.

Lothian, J. R., and C. H. McCarthy. 2001. "Equity Returns and Inflation: The Puzzlingly Long Lags." International Finance 0107003, EconWPA.

Ludwig, A., and T. Sløk. 2004. "The Relationship Between Stock Prices, House Prices and Consumption in OECD Countries." The B.E. Journal of Macroeconomics 4: 1-28.

Mitchell, B. 1998. International Historical Statistics. New York: Stockton Press.

Modigliani, F., and R. A. Cohn. 1979. "Inflation, Rational Valuation and the Market." Financial Analyst Journal 35: 22-44.

O’Donoghue, J., L. Coulding, and C. Allen. 2004. “Consumer Price Inflation since 1750.” Economic Trends 604: 38-46.

Rua, A., and L. C. Nunes. 2009. "International Comovement of Stock Market Returns: A Wavelet Analysis." Journal of Empirical Finance 16: 632639.

Schotman, P. C., and M. Schweitzer. 2000. “Horizon Sensitivity of the Inflation Hedge of Stocks.” Journal of Empirical Finance 7: 301-305.

Simo-Kengne, B., S. Miller, R. Cupta, and G. Aye. 2015. "Time-Varying Effects of Housing and Stock Returns on US Consumption." The Journal of Real State Finance and Economics 50: 339-354.

Solnik, B., and V. Solnik. 1997. A Multi-Country Test of the Fisher Model for Stock Returns." Journal of International Financial Markets, Institutions and Money 7: 289-301.

Tiwari, A. K. 2013. “Oil Prices and the Macroeconomy Reconsideration for Cermany: Using Continuous Wavelet.” Economic Modelling 30: 636642.

Torrence, C., and C. P. Compo. 1998. “A Practical Guide to Wavelet Analysis.” Bulletin of the American Meteorological Society 79: 605-618.

Torrence, C., and P. Webster. 1999. "Interdecadal Changes in the Esnom on Soon System." Journal of Climate 12: 2679-2690.

Tsong, C. C., and C. F. Lee. 2013. “Quantile Cointegration Analysis of the Fisher Effect." Journal of Macroeconomics 35: 186-198.

Valcarcel, V. J. 2012. “The Dynamic Adjustments of Stock Prices to Inflation Disturbances.” Journal of Economics and Business 64: 117-144.

Supplementary Material: The online version of this article offers supplementary material (DOI: https://doi.org/10.1515/snde-2017-0049). 